mich doch nicht entschliefsen, diese Tiere noch in die unmittelbare Verwandtschaft der gallica zu stellen, da die Punktierung des ersten Abdominalsegments mit der der thoracica übereinstimmt, eher noch etwas dichter ist und nicht die für die gallica charakteristische Grundchagrinierung erkennen läfst. Die Entscheidung darüber, ob, wir es hier mit einer neuen, der gallica nahestehenden Art oder einer extremen Form derselben zu tun haben, kann erst die Kenntnis des zugehörigen Weibchens bringen. Als eine der gallica enger verwandte Art ist auch die infirma Mor. anzusprechen.

\title{
Ein Beitrag zur Kenntnis der Pflanzengallen der Provinz Brandenburg.
}

Von H. Ross, München-Nymphenburg.

Da man seit einiger Zeit bestrebt ist, Vorkommen und Verbreitung der Pflanzengallen in der Provinz Brandenburg festzustellen, veröffentliche ich nachfolgenden kleinen Beitrag in der Hoffnung, dafs derselbe der Sache nützen wird. Es bot sich mir Gelegenheit, Gallen in der Mark zu sammeln, da ich mehrfach meinen Urlaub bei dort ansässigen Verwandten verbrachte. Hauptsächlich weilte ich in Strehlow (abgekürzt St.) in der Uckermark, $10 \mathrm{~km}$ südlich von Prenzlau, sowie in Clessin (abgekürzt Cl.), $13 \mathrm{~km}$ nördlich von Frankfurt a. O. Einige wenige Beobachtungen machte ich aufserdem gelegentlich der Versammlung der Deutschen Dendrologischen Gesellschaft in Eberswalde (abgekürzt Eb.) im August 1920. Das Belegmaterial habe ich dem Gallenherbar der Provinz Brandenburg im Botanischen Museum, Berlin-Dahlem, übergeben.

Die den Gallen beigefügten Nummern beziehen sich auf mein Buch „Die Pflanzengallen Mittel- und Nordeuropas"; dadurch wird eine Beschreibung der einzelnen Gallen entbehrlich.

Gallmilbe.

Acer platanoides $\mathrm{L}$.

$\mathrm{Cl}$.

Acer pseudoplatanus $\mathrm{L}$.

(26) Eriophyes pseudoplatani Corti. - St.

(31) Eriophyes macrochelus typicus Nal. - St.

(64) Blattlaus. - Cl.

\section{Aegopodium podagraria $\mathbf{L}$.}

\section{Aseseculus hippocastanum $\mathbf{L}$.}

(68) Eriophyes hippocastani (Fock.). - Cl.

Alnus glutinosa Gaertn.

(112) Eriophyes brevitarsus typicus (Fock.). - St. 


\section{Alnus incana Willd.}

(103) Eriophyes laevis alni incanae Nal. - St.

\section{Artemisia campestris $\mathrm{L}$.}

(181) Boucheella artemisiae (Bché.). - St., Cl., Eb.

\section{Artemisia vulgaris $\mathrm{L}$.}

(196) Cryptosiphum artemisiae Pass. - Cl.

(199) Eriophyes artemisiae (Can.). - Cl.

\section{Atriplex patulum L.}

(234) Aphis atriplicis L. - St., Cl.

\section{Berteroa incana DC.}

(264) Ceuthorrhynchus pleurostigma Marsh. - Cl.

Campanula (?trachelium Lo).

(364) Eriophyes schmardai Nal. - Cl.

\section{Crataegus oxyacantha $\mathbf{L}$.}

(527) Dasyneura crataegi (Winn.). - Beim Bahnhof Karzig, nördlich von Frankfurt (Oder).

(534) Eriophys goniothorax Nal. - St.

Echium vulgare $\mathrm{L}$.

(588) Eriophyes echii (Can.). - Cl.

\section{Euphorbia cyparissias $\mathbf{L}$.}

(631) Bayeria capitigena (Br.). - Cl.

\section{Evonymus europaea $\mathrm{L}$.}

(641) Eriophyes convolvens Nal. - Cl.

Fagus silvatica L.

(659) Eriophyes stenaspis typicus Nal. - $\mathrm{Cl}$.

\section{Fraxinus excelsior L.}

(688) Eriophyes fraxinivorus Nal. - Cl.

(694) Dasyneura fraxini (Kieff.). - Cl.

Galium aparine L.

(708) Dasyneura aparines (Kieff.) + Macrolabis jaapi Rübs. - St.

\section{Galium mollugo $\mathbf{L}$.}

(718) Eriophyes galiobius (Can.). - St., in der Sandgrube.

(733) Aphis galii Kalt. - St.

\section{Galium parisiense L.}

(711) Dasyneura spec. - St.

\section{Galium verum $\mathbf{L}$.}

(734) Aphis bicolor Koch. -- St., Cl. 
Ross, Ein Beitr. z. Kenntn. d. Pflanzengallen der Prov. Brandenburg. 293

\section{Juglans regia $\mathrm{L}$.}

(882) Eriophyes tristriatus Nal. - $\mathrm{Cl}$.

(883) Eriophyes tristriatus erineus Nal. - $\mathrm{Cl}$.

\section{Medicago falcata L., sativa $\mathrm{L}$.}

(1043) Dasyneura ignorata (Wachtl). - St. und beim Bahnhof Seehausen (Uckermark).

(1051) Jaapiella medicaginis (Kieff.). - St.

\section{Mentha aquatica L.}

(1061) Eriophyes megacerus Can. et Massal. - St.

Papaver rhoeas $\mathrm{L}$.

(1123) Aylax minor Htg. - St.

Picea excelsa Lk.

(1161) Chermes abietis Kalt. - Cl.

(1162) Cnaphalodes strobilobius (Kalt.). - Cl.

\section{Pirus communis L.}

(1189) Eriophyes piri (Pagenst.). - Cl.

\section{Populus nigra $\mathbf{L}$.}

(1277) Pemphigus spirothecae Pass. - Cl.

(1281) Pemphigus filaginis (Fonsc.) (Frühjahrsgeneration). - Cl.

\section{Prumus domestica $\mathbf{L}$.}

(1336) Eriophyes similis Nal. - $\mathrm{Cl}$.

\section{Prunus spinosa L.}

(1326) ? Dasyneura tortrix F. Lw. - Cl.

(1332) Putoniella marsupialis F. Lw. - Cl.

(1336) Eriophyes similis Nal. - Cl.

(1349) ? Phorodon humuli (Schrk.). - Cl.

\section{Quercus robur $\mathbf{L}$.}

(1363) Biorrhiza pallida (01.) or․ - Cl.

(1435) Andricus ostreus Htg. 우. - Cl., Eb.

(1437) Diplolepis quercus-folii (L.) 우. - Cl.

(1439) Diplolepis longiventris (Htg.) 웅 - $\mathrm{Cl}$.

(1444) Neuroterus numismalis (Fourc.) $q$ q. - Eb.

(1445) Neuroterus albipes laeviusculus (Schck.) 웅 - $\mathrm{Cl}$.

(1447) Neuroterus quercus-baccarum lenticularis (0l.) 웅 - Cl., Eb.

(1465) Macrodiplosis dryobia (F. Lw.), - Cl.

\section{Raphanus raphanistrum $\mathrm{L}$.}

(1567) Gephyraulus raphanistri (Kieff.). - $\mathrm{Cl}$.

\section{Rhamnus cathartica $\mathbf{L}$.}

(1576) Trichochermes walkeri (Först.). - $\mathrm{Cl}$.

\section{Robinia pseudacacia L.}

(1599, 1600) Phyllocoptes robiniae Nal. + allotrichus Nal. - Cl. 


\section{Roripa silvestris Rehb.}

(1085) Dasyneura sisymbrii (Schrk.). - St., Cl.

Rosa spec. div.

(1602) Rhodites rosae (L.). - Cl.

(1608) Rhodites eglanteriae $\mathrm{Htg}$. - $\mathrm{Cl}$.

(1611) Wachtliella rosarum (Hardy). - Cl.

Salix alba L.

(1644) 'Phyllocoptes parvus Nal. - St. Wirrzöpfe aus Blütenkätzchen und aus vegetativen Sprossen.

(1663) Rhabdophaga terminalis (H. Lw. $)^{1}$ ). - St.

(1664) Rhabdophaga rosaria (H. Lw.). - Cl.

Salix alba L. $\times$ fragilis $\mathrm{L}$.

(1663) Rhabdophaga terminalis (H. Lw.). - St.

Saliac cinerea $\mathbf{L}$.

(1690) Iteomyia capreae major (Kieff.). - St.

Salia fragilis $\mathrm{L}$.

(1696) Pontania capreae (L.). - St.

Saliax purpurea L.

(1698) Pontania viminalis (L.). - Cl.

Salia triandra L.

(1663) Dasyneura terminalis (H. Lw.). - Cl.

Salia viminalis L.

(1709) Rhabdophaga marginemtorquens (Winn.). - Cl.

Sambucus nigra $\mathbf{L}$.

(1719) Epitrimerus trilobus Nal. - St., Cl.

Sisymbrium sophia $\mathrm{L}$.

(1822) ? Dasyneura sisymbrï (Schrk.). - St.

Sonchus asper Gars.

(1839) Cystiphora sonchi (F. Lw.). - St., Cl.

Stachys silvatica $\mathbf{L}$.

(1846) Wachtliella stachydis (Br.). - St.

\section{Syringa vulgaris $\mathrm{L}$.}

(1876) Eriophyes löwi Nal. - Cl.

Tilia cordata Mill.

(1925) Eriophyes tetratrichus typicus Nal. - Cl.

(1927) Eriophyes tiliae rudis Nal. - St., Cl.

1) Nach brieflicher Mitteilung von Rübsaamen an Hedicke gehört diese in der Literatur als Dasyneura bezeichnete Spezies zu Rhabdophaga. 
(1931) Eriophyes tiliae liosoma Nal. - Cl. Auch auf den Hochblättern.

Tilia platyphyllos Scop.

(1924) Dasyneura tiliamvolvens Rübs. - St.

\section{Ulmus campestris L.}

(1984) Brysocrypta pallida (Hal.). - Cl.

(1987) Schizoneura ulmi (L.). - Cl.

(1990) Tetraneura ulmi (Deg.). - Cl.

\section{Veronica chamaedrys L.}

(2025) Jaapiella veronicae (Vall.). - St., Cl.

Viola tricolor L. var. arvensis Murr.

(2080) Dasyneura violae (F. Lw.). - St., Cl.

Einige neue Drosophiliden.

Von Dr. Günther Enderlein, Berlin.

Zaprionus vittiger Coqu. 1901.

Die 3 Orbitalborsten jederseits im inneren schwarzen Saum der weifsen Längsstriemen der Stirn. Fühler rostgelb. Rückenschild mit 4 feinen, weifsen, schwarzgesäumten Längsstriemen; die beiden mittelsten laufen bis in die hinteren Seitenecken des Scutellums. Spitze des Scutellums ohne weifsen Fleck. Abdomen einfarbig matt rostgelblich. 2. Medianabschnitt $2^{1} / 2$ der mcu-Querader.

Diese Species liegt von folgenden Lokalitäten vor:

Ost-Afrika, Nyassasee, L a n g e n b u r g. 1898. Gesammelt von Professor Dr. F ülle born.

Ost-Afrika, Kiungani. Gesammelt von M e i n h of.

\section{Zaprionis albicornis nov. spec.}

Die Unterschiede von $Z$. vittiger sind:

3. Fühlerglied ist lebhaft weifs. Die 3 Orbitalborsten jederseits am Innenrand der 2 weifsen Seitenstriemen der Stirn dicht am Augenrand. Die beiden mittleren weilsen Längsstreifen des Rückenschildes setzen sich nur bis in die vorderen Seitenecken des Scutellums fort. Die seitlichen weifslichen Längsstreifen fehlen völlig, nur die Schulterbeulen sind etwas weifslich aufgehellt. Spitze des Scutellums mit rundlichem weifsen Fleck. Die Abdominaltergite mit Ausnahme des Vorderdrittels und bei dem 4. und 5. Tergit auch noch mit Ausnahme der Medianlinie dunkelbraun. $\mathrm{r}_{4}+5$ und $\mathrm{m}$ parallel. 2. Medianabschnitt doppelt so lang wie die mcu-Querader.

Körperlänge $2 \mathrm{~mm}$.

Formosa, Toyenmongoi bei Tainan. 1910. (Rolle V.). 


\section{$2 \mathrm{BHL}$ Biodiversity Heritage Library}

Ross, H . 1922. "Ein Beitrag zur Kenntnis der Pflanzengallen der Provinz Brandenburg." Deutsche entomologische Zeitschrift 1922(3), 291-295. https://doi.org/10.1002/mmnd.48019220304.

View This Item Online: https://www.biodiversitylibrary.org/item/103380

DOI: https://doi.org/10.1002/mmnd.48019220304

Permalink: https://www.biodiversitylibrary.org/partpdf/236302

\section{Holding Institution}

Harvard University, Museum of Comparative Zoology, Ernst Mayr Library

\section{Sponsored by}

Biodiversity Heritage Library

\section{Copyright \& Reuse}

Copyright Status: Public domain. The BHL considers that this work is no longer under copyright protection.

This document was created from content at the Biodiversity Heritage Library, the world's largest open access digital library for biodiversity literature and archives. Visit BHL at https://www.biodiversitylibrary.org. 